\title{
SÍFILIS CONGÊNITA: UMA ANÁLISE DO PERFIL EPIDEMIOLÓGICO NO MUNICÍPIO
}

\author{
DE CRICIÚMA ${ }^{1}$
}

\author{
Luana Rosso ${ }^{2}$ \\ Mariá Vaz Franco Martins ${ }^{3}$ \\ Maria Tereza Soratto 4 \\ Ivanir Prá da Silva Thomé ${ }^{5}$ \\ Rosilda Lopes de Souza ${ }^{6}$ \\ Recebido em: 01 dez. 2017 \\ Aceito em: 19 dez. 2018
}

RESUMO: A sífilis é uma doença infecciosa sistêmica de evolução crônica e muitas vezes assintomática, transmitida principalmente por via sexual e vertical. O estudo em questão trata-se de um estudo de corte transversal, retrospectivo, descritivo de natureza quantitativa e documental, a coleta de dados se deu no Programa de Atenção Municipal as DST/HIV/AIDS (PAMDHA) do município de Criciúma, através de pesquisa documental com base nos dados da Vigilância Epidemiológico do SINAN com o objetivo de investigar os casos de sífilis congênita no município. Através da pesquisa pode-se perceber que entre os anos de 2015 e 2016 constatou 3,7\% do total de casos pesquisados chegaram a óbito, dos RN nascidos com sífilis $51,9 \%$ foram assintomáticos e 3,8\% sintomáticos, os demais não apresentaram características. Do total de gestantes, $66,6 \%$ realizou pré-natal e foram diagnosticadas a partir do mesmo, possibilitando o tratamento. Para que haja uma menor prevalência de casos de sífilis é necessário que os profissionais da saúde adotem, além das políticas públicas já disponíveis outros hábitos que levem ao alcance de todas as classes de gestantes, para evitar mais casos de sífilis.

Palavras-chave: Gestantes. Sífilis Congênita. Recém-Nascidos. Infecções Sexualmente Transmissíveis.

\section{CONGENITAL SYPHILIS: AN ANALYSIS OF THE EPIDEMIOLOGICAL PROFILE IN}

\section{THE CITY OF CRICIÚMA}

ABSTRACT: Syphilis is a systemic infectious disease of chronic evolution and often asymptomatic, transmitted mainly through sexual and vertical. The study in question it is a cross-sectional study, retrospective, descriptive quantitative and documentary nature, data collection took place in the Attention the Municipal STD/HIV/AIDS (PAMDHA) of the city of Criciúma, through documentary research based on Epidemiological Surveillance data from SINAN aiming to investigate the cases of congenital syphilis in the municipality. Through research you can notice that between the years 2015 and 2016 found $3.7 \%$ of total cases surveyed came to death, the RN born with syphilis $51.9 \% 3.8 \%$ were asymptomatic and symptomatic, the others did not

\footnotetext{
${ }^{1}$ Artigo baseado no Trabalho de Conclusão do Curso de Enfermagem - UNESC.

2 Enfermeira - UNESC- Criciúma - SC - Brasil. E-mail: luh.rosso@hotmail.com.

${ }^{3}$ Enfermeira - UNESC-Criciúma - SC - Brasil. E-mail: maria_martinsss@hotmail.com.

${ }^{4}$ Enfermeira - Mestre - UNESC- Criciúma - SC - Brasil. E-mail: guiga@unesc.net.

${ }^{5}$ Enfermeira - Mestre - UNESC - Criciúma - SC - Brasil. E-mail: ivanir_pdst@yahoo.com.br.

${ }^{6}$ Enfermeira - Mestre- UNESC - Criciúma - SC - Brasil. E-mail: rozildalopes@unesc.net.
} 
show characteristics. Of the total of $66.6 \%$ pregnant women, prenatal and conducted were diagnosed from the same, allowing the treatment. So there is a lower prevalence of syphilis cases is necessary that health professionals adopt, in addition to the already available public policies other habits that lead to reach of all classes of pregnant women, to prevent more cases of syphilis.

Keywords: Pregnant women. Congenital Syphilis. Newborns. Sexually Transmitted Infections.

\section{INTRODUÇÃO}

As Infecções Sexualmente Transmissíveis (IST), anteriormente designadas doenças venéreas, são infecções que se transmitem por contato sexual. São causadas por diversos agentes infecciosos e ocasionam grande multiplicidade de sintomas e manifestações clínicas, embora, na maioria dos casos, possam evoluir com pouco ou nenhum sintoma (AZEVEDO, 2008).

As IST constituem atualmente um problema de saúde pública a nível mundial, com um peso socioeconômico crescente, não só pelo elevado número de pessoas infectadas e pelo aumento da incidência em muitos países, mas, sobretudo pelas suas consequências em nível da saúde sexual, reprodutiva e materno-fetal e, ainda, pela sua capacidade de facilitar a transmissão (AZEVEDO, 2008).

As Infecções sexualmente transmissíveis (IST), por lidarem com as questões ligadas à sexualidade, tendem a ser encobertas, tornarem-se invisíveis. No caso da sífilis, pela remissão espontânea das lesões das fases primária e secundária, seguida de longo período de latência clínica, fica difícil a identificação de portadores, levando Carrara (1996, p.46) a dizer que "se a sífilis se propaga tão amplamente é em grande parte por ser invisível".

A sífilis é uma doença infecciosa sistêmica causada pelo vírus Treponema Pallidum, de evolução crônica e muitas vezes assintomática, que tem como principais formas de transmissão às vias sexual e vertical. Exclusiva do ser humano, e quando não tratada precocemente, pode evoluir para uma enfermidade crônica com sequelas irreversíveis em longo prazo (BRASIL, 2010). Não existe vacina contra a sífilis, e a infecção pela bactéria causadora não confere imunidade protetora. Isso significa que as pessoas poderão ser infectadas tantas vezes quantas forem expostas ao $T$. pallidum (ORGANIZAÇÃO MUNDIAL DA SAÚDE, 2015).

A forma adquirida da sífilis subdivide-se em precoce e tardia, dependendo do tempo de infecção e do grau de infecção. A sífilis congênita apresenta-se de forma variável, desde assintomática, em 70\% dos casos, até formas mais graves (BRASIL, 2007).

A sífilis congênita é o resultado da disseminação hematogênica do Treponema pallidum, da gestante infectada não tratada ou inadequadamente tratada para o seu concepto, por via transplacentária. Os principais fatores que determinam a probabilidade de transmissão vertical do T. pallidum são o estágio da sífilis na mãe e a duração da 
exposição do feto no útero (BRASIL, 2006).

A síndrome clínica da sífilis congênita precoce surge até o segundo ano de vida e deve ser diagnosticada por meio de uma avaliação epidemiológica criteriosa da situação materna e de avaliações clínicas, laboratorial e de estudos de imagem na criança. Entretanto, o diagnóstico na criança representa um processo complexo (BRASIL, 2006).

A síndrome clínica da sífilis congênita tardia surge após o $2^{\circ}$ ano de vida, os sinais e sintomas podem ser tíbia em "lâmina de sabre"; fronte olímpica; nariz em sela; dentes incisivos medianos superiores deformados (dentes de Hutchinson); mandíbula curta; arco palatino elevado; ceratite intersticial; surdez neurológica; dificuldade no aprendizado (BRASIL, 2007). Da mesma forma que a sífilis congênita precoce, o diagnóstico deve ser estabelecido por meio da associação de critérios epidemiológicos, clínicos e laboratoriais. Além disso, deve-se estar atento na investigação para a possibilidade de acriança ter sido exposta ao T. pallidum por meio de exposição sexual (BRASIL, 2006).

No mundo, observa-se que a sífilis é uma infecção reemergente, como verificado na Itália e nos Estados Unidos da América, chamando a atenção para a necessidade de rastreamento para todas as gestantes durante o pré-natal e tratamento em tempo hábil, com o objetivo de conter a infecção congênita (TRIDAPALLI, 2012). Na América Latina, na África e em países da Ásia permanece elevada sua incidência e seu controle tem como foco a assistência pré-natal (VALDEMARRA et al., 2004).

No Brasil, segundo o Ministério da Saúde (MS), ao ano, 50 mil parturientes têm o diagnóstico de sífilis, com prevalência variando de 1,1 a 11,5\%, em função da assistência pré-natal e do grau de instrução materna. Em um estudo realizado em 2014, obteve-se um resultado de aproximadamente 12 mil nascidos vivos têm sífilis congênita no Brasil por ano (LORENZI; FIAMINGHI; ARTICO, 2009).

Segundo dados do Boletim Epidemiológico de 2016 a sífilis adquirida teve um aumento de $32,7 \%$, a sífilis em gestantes 20,9\% e congênita de 19\%. Em 2015 o número total de casos notificados de sífilis no Brasil foi de 65.878, no mesmo período a taxa de detecção foi de 42,7 casos por 100 mil habitantes (BRASIL, 2016).

Em gestantes, no ano de 2015, a taxa de detecção de sífilis foi de 11,2 casos a cada 1.000 nascidos vivos, considerando o total de casos da doença. Com relação à Sífilis Congênita em 2015 foi notificado 19.228 casos da doença, uma taxa de incidência de 6,5 por 1.000 nascidos vivos (BRASIL, 2016).

O tratamento para sífilis congênita é realizado com penicilina conforme os critérios determinados pelo Ministério da Saúde. Níveis de penicilina superiores a $0,018 \mathrm{mg} / \mathrm{kg}$ são considerados suficientes e devem ser mantidos por pelo menos sete a 10 dias na sífilis recente, e por duração mais longa na sífilis tardia (BRASIL, 2015b).

Sendo assim, é de grande importância fazer o teste para detectar a sífilis durante o pré-natal, pois se o resultado é positivo, deve-se iniciar o tratamento adequado (da mulher e seu parceiro) para evitar a transmissão da doença para o bebê. Entretanto vale lembrar 
que o uso do preservativo nas relações sexuais ainda é a melhor forma de prevenção desta e outras doenças sexualmente transmissíveis.

A partir disso deu-se como objetivo geral analisar o perfil epidemiológico da sífilis congênita no município de Criciúma entre os anos de 2015 e 2016.

\section{MATERIAL E MÉTODOS}

O estudo caracterizou-se como quantitativo, de corte transversal, retrospectivo, descritivo e documental, realizado no curso de Enfermagem da Universidade do Extremo Sul Catarinense - UNESC.

Os dados do estudo foram coletados no Programa de Atenção Municipal às DST/HIV/AIDS (PAMDHA), tendo como base de dados o Sistema de Informações de Agravos de Notificação (SINAN) e o prontuário eletrônico, tendo como população diagnosticadas e notificadas no SINAN e no PAMDHA com Sífilis, no ano de 2015 e 2016, residentes do município de Criciúma/SC.

Para a análise estatística foi montada uma planilha eletrônica para elaboração do banco de dados a partir da coleta de dados realizada nesta pesquisa. As variáveis quantitativas foram apresentadas em média \pm desvio padrão, para todas as análises estatísticas foi adotado $o$ valor de $p<0,05$ para indicar diferença estatisticamente significativa entre os grupos. As análises foram realizadas no Microsoft Office Excel.

Para a realização da pesquisa foi assinado um termo de confidencialidade, sendo que este assegura o sigilo das informações da pesquisa. O termo segue as exigências formais contidas na resolução 466/12 e 510/16, do Conselho Nacional de Saúde.

\section{RESULTADOS E DISCUSSÃO}

No período de 2015 e 2016 foram notificados 27 casos de gestantes com sífilis no município de Criciúma, sendo utilizados para estudo os casos notificados no SINAN e dados contidos no prontuário eletrônico dos pacientes.

No primeiro gráfico é verificado o número de óbitos fetais e natimorto por sífilis neonatal. 
Gráfico 1 - Óbitos Fetais causados por Sífilis Congênita

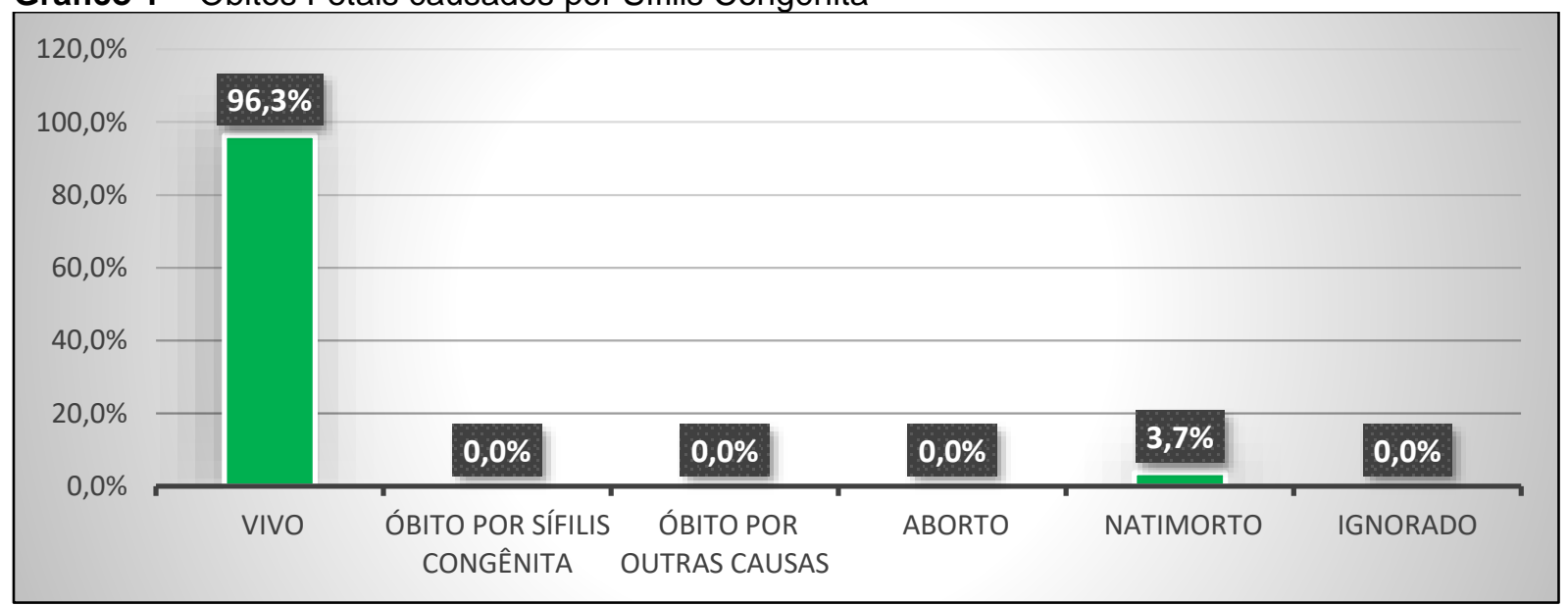

Fonte: Vigilância Epidemiológica, 2015-2016.

De acordo com os dados encontrados junto da Vigilância Epidemiológica do município de Criciúma/SC e com base nos dados do SINAN foi possível observar no gráfico um que entre os anos de 2015 e 2016 o percentual de recém-nascidos natimortos foi de $3,7 \%$ e vivos $96,3 \%$.

Steer et al. (1989) em seus estudos traz que cerca de $15 \%$ do total das gestantes apresentam abortos espontâneos no primeiro trimestre da gravidez e, para gestantes com sífilis, estima-se que as perdas sejam maiores.

Temmerman et al. (1992) demonstraram, em estudo caso-controle, uma associação entre aborto espontâneo e sorologia materna reativa para sífilis, com um risco 4,3 vezes maior de ocorrência de abortamento nas mulheres com sífilis, mesmo controlando para outros fatores. Resultados diferentes foram mostrados por Labbe et al. (2002) na Guiné- Bissau, em estudo caso-controle, demonstrando forte associação de sorologia positiva para sífilis com natimortalidade e parto prematuro, porém, não com abortamento espontâneo.

No segundo gráfico são verificadas as características dos Recém-Nascidos com Sífilis Congênita.

Gráfico 2 - Características do Recém-Nascido com Sífilis Congênita

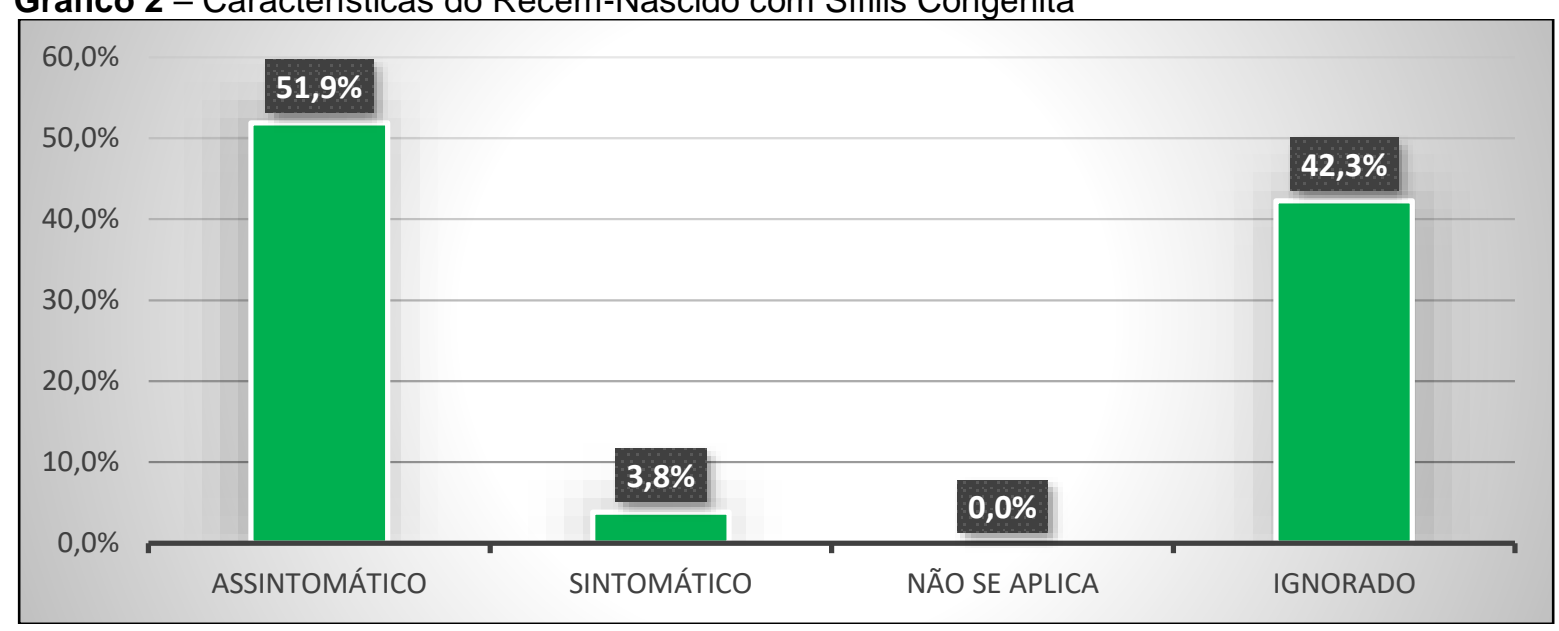

Fonte: Vigilância Epidemiológica, 2015-2016. 
Destes $96,3 \%$ nascidos vivos e diagnosticados com Sífilis Congênita, $51,9 \%$ foram caracterizados assintomáticos 3,8\% sintomáticos e $42,3 \%$ não foi identificada nenhuma característica, registrando como ignorados, conforme mostra o gráfico dois.

Estudos de Magalhães et al. (2013), trazem que entre cinquenta recém-nascidos, $3(6 \%)$ apresentaram ao nascimento sinais clínicos de infecção congênita por sífilis, o que podemos comparar com nossa pesquisa, onde $51,9 \%$ foram caracterizados com assintomáticos, ou seja, não apresentaram características de sífilis.

Nos estudos de Domingues et al. (2013), quatro recém-nascidos eram assintomáticos ao nascimento e não havia registro do diagnóstico clínico ou de sinais e sintomas da doença na ficha de investigação em sete casos.

A maioria das fichas de notificação de sífilis em gestantes e seus Recém Nascidos estavam incompletos, não contendo na maioria dados importantes como o tratamento das gestantes e características dos recém-nascidos, deve-se realizar um treinamento para que sejam sempre preenchidas corretamente as fichas de notificação, para que futuramente possa colaborar com os dados epidemiológicos, podendo melhorar os resultados.

No terceiro gráfico podem-se observar os sinais e sintomas dos Recém-Nascidos com sífilis congênita.

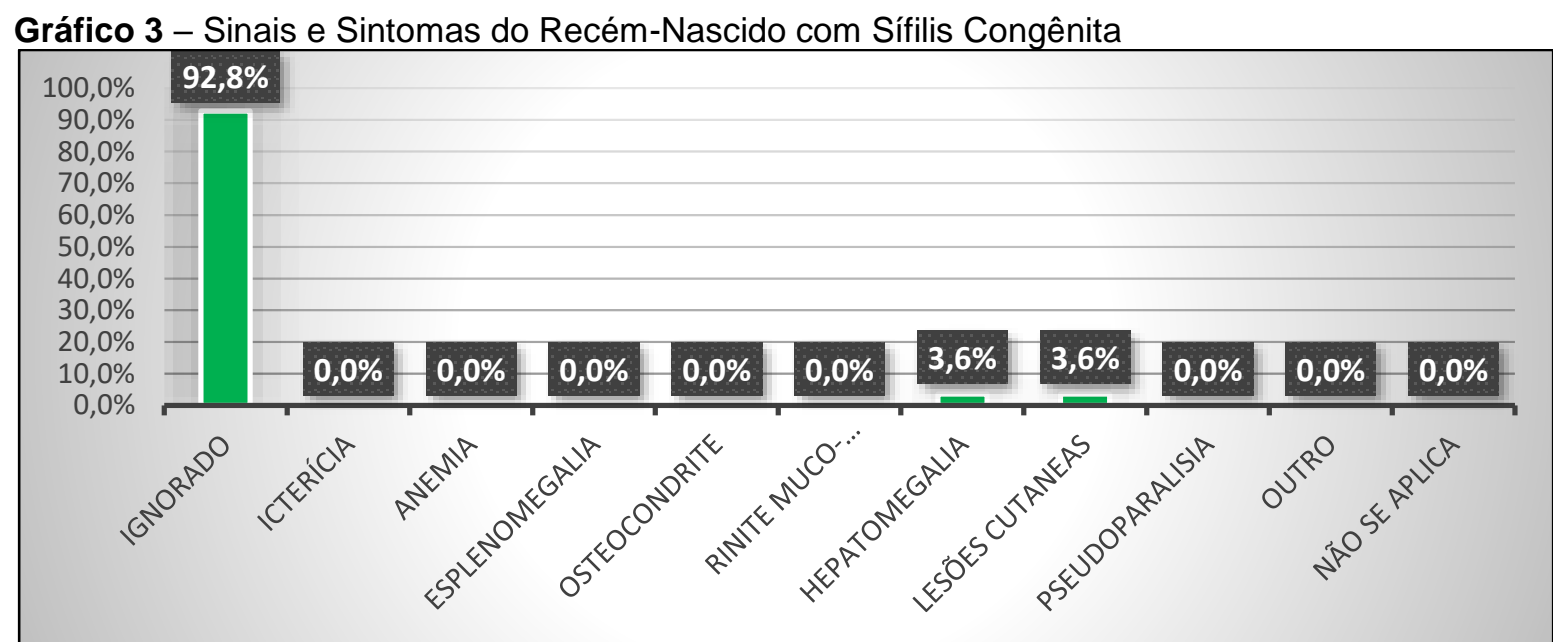

Fonte: Vigilância Epidemiológica, 2015-2016.

O gráfico três traz os principais sinais e sintomas relatados entre os recém-nascidos com Sífilis Congênita, como apresentado acima $92,8 \%$ dos dados não constavam os sinais ou sintomas, sendo registrados como ignorados, $3,6 \%$ apresentaram hepatomegalia $\mathrm{e}$ outros 3,6\% apresentaram lesões cutâneas.

Sobre o número de casos notificados de sífilis congênita, cabe esclarecer que o Brasil adotava um modelo sensível. O critério de definição de casos englobava praticamente todas as crianças que nasciam de mães que houvessem sido diagnosticadas com a doença, mesmo que a gestante tivesse sido tratada, por falta de informações de tratamento de seus parceiros (BRASIL, 2015a).

Quando realizada a coleta de dados, todas as pacientes constavam em um 
prontuário eletrônico onde estava descrito se haviam realizado ou não seus tratamentos, destes prontuários a maioria estava incompleto, ou seja, observamos que apenas uma pequena parcela havia realizado o tratamento. É necessário que seja sempre notificado todos os tratamentos realizados no prontuário, para que haja um melhor controle da sífilis.

No gráfico quatro verificamos o momento de diagnóstico de sífilis materna.

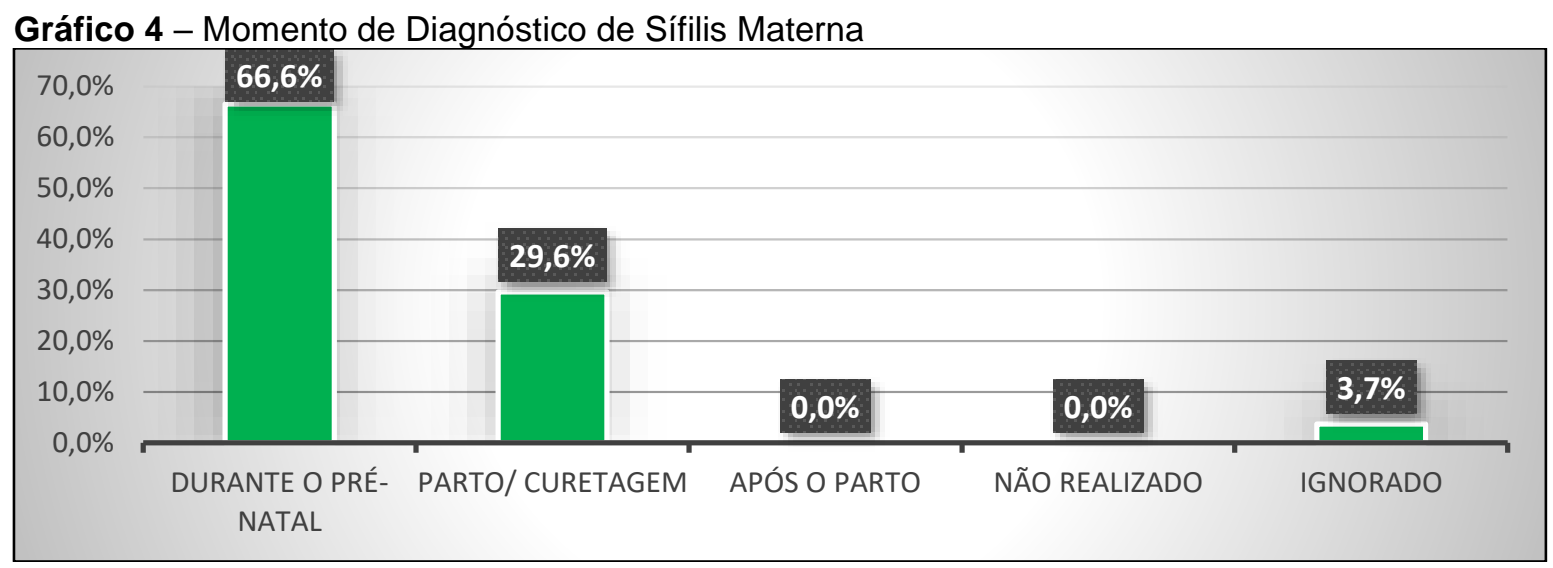

Fonte: Vigilância Epidemiológica, 2015-2016.

No quarto gráfico apresentamos os dados referentes ao momento do diagnóstico de Sífilis Materna, 66,6\% das mulheres foram diagnosticadas ainda no pré-natal, 29,6\% foram diagnosticadas somente no parto ou na curetagem e apenas 3,7\% não foram encontrados nenhuma característica, registrando como ignorado.

Comparando os nossos resultados com o estudo de Saraceni e Leal (2003), nesse estudo todos os casos confirmados e mesmo nas que tiveram o diagnóstico próximo ao parto, foi maior o percentual de identificação prévia da doença. Da mesma forma, a positividade do VDRL no momento do parto foi significativamente menor, refletindo a eficácia do tratamento.

No gráfico cinco verificamos a realização de pré-natal durante a gestação.

Gráfico 5 - Realização de Pré-Natal na Gestação

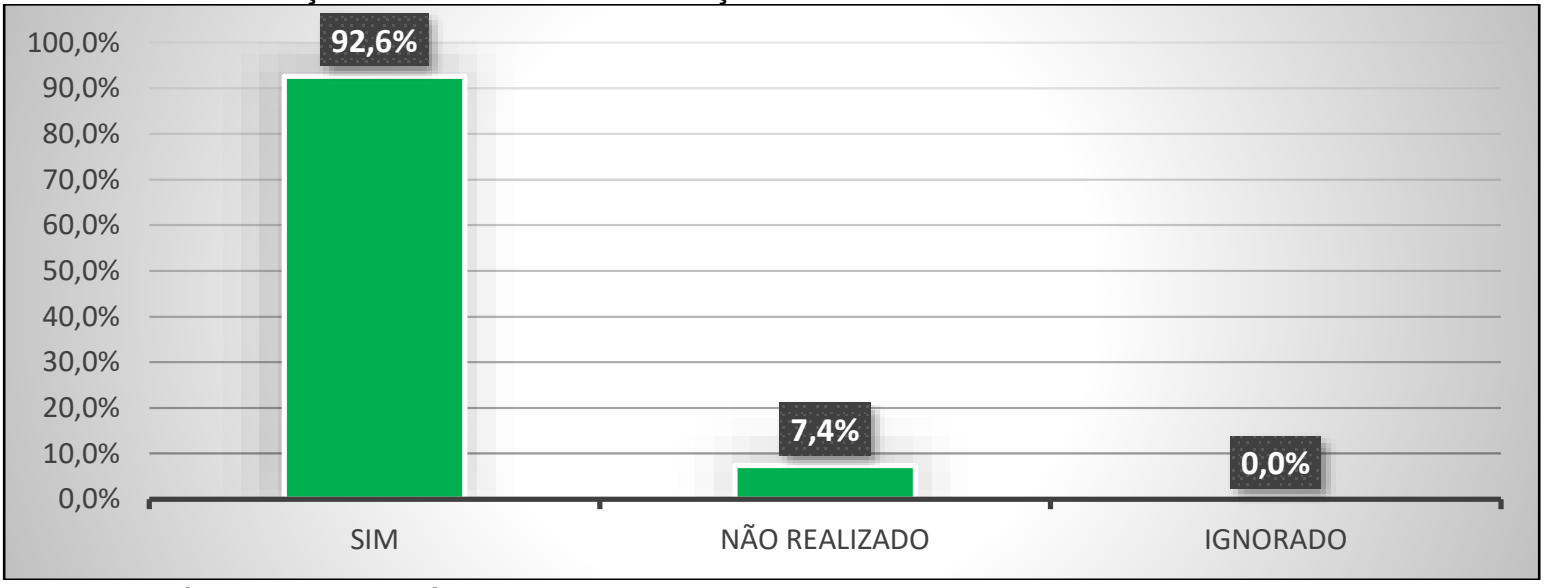

Fonte: Vigilância Epidemiológica, 2015-2016.

De acordo com o quinto gráfico, dos dados obtidos a partir da pesquisa realizada 
na Vigilância Epidemiológica do município pode-se observar que 92,6\% das gestantes realizaram o pré-natal durante a gestação e apenas 7,4\% não realizaram o pré-natal.

Em relação ao acesso ao pré-natal, segundo dados do Ministério da Saúde em $2015,78,4 \%$ das mães de crianças com sífilis congênita fizeram pré-natal, enquanto 15,0\% não fizeram, e 6,7\% apresentaram informação ignorada. Entre aquelas que fizeram o prénatal, 51,4\% tiveram diagnóstico de sífilis durante o pré- natal, 34,6\% no momento do parto/curetagem, 8,3\% após o parto e 0,6\% não tiveram diagnóstico, além de 5,1\% constarem como ignorados. Com relação ao esquema de tratamento da gestante, 56,5\% receberam tratamento inadequado, $27,3 \%$ não receberam tratamento, e apenas $4,1 \%$ receberam tratamento adequado (BRASIL, 2016).

Os achados, corroborando com os dados de Camargo e Coeli (2000), permitem inferir sobre a maneira como profissionais de saúde se comportam frente ao preenchimento de instrumentos de notificação, levando em consideração a importância creditada a determinado evento ou situação pelo médico assistente, que pode registrar ou não certos dados. A ficha de notificação de Sífilis Congênita, como as demais fichas que compõem o sistema de vigilância epidemiológica, tem instrução para preenchimento em anexo, que pode não estar sendo suficiente para esclarecer sobre o correto preenchimento da mesma.

Os benefícios do tratamento para sífilis se estendem para a redução da morbidade e da mortalidade relacionadas à sífilis nos recém-nascidos. As reduções dos índices de prematuridade, natimortalidade e neomortalidade foram significativas no grupo captado (SARACENI; LEAL, 2003), o que corrobora com nosso estudo, onde foi observada uma pequena taxa de natimortos. Embora a sífilis seja uma doença para a qual existem recursos diagnósticos e terapêuticos simples e de baixo custo, seu controle na gestação mostra-se um desafio para profissionais de saúde e gestores. Isso em decorrência do curto intervalo da gestação para a realização do seu diagnóstico e tratamento; pela dificuldade de abordagem das doenças sexualmente transmissíveis, principalmente durante a gestação; e provavelmente pelo desconhecimento da magnitude desse agravo e dos danos que ele pode causar à saúde da mulher e do bebê pela população e pelos profissionais de saúde (DOMINGUES et al., 2013).

A maior prevalência de sífilis na gestação em mulheres de baixa condição socioeconômica, com antecedentes obstétricos de risco e pior acesso a serviços de 30 saúde, indica a maior vulnerabilidade social e reprodutiva dessas mulheres que tornam mais complexo o desafio de controle da sífilis nessa população (DOMINGUES et al., 2013).

Em 2014, um dos critérios de definição de casos foi alterado: a ausência de informação de tratamento do parceiro sexual da mãe deixa de ser um dos critérios de notificação de sífilis congênita, mantendo-se apenas os casos de parceiro(s) com sífilis sabidamente não tratada (BRASIL, 2015a).

Estratégias inovadoras são necessárias, visando: à captação precoce das gestantes para o início da assistência pré-natal no primeiro trimestre gestacional; à garantia do diagnóstico da doença durante a gestação no menor prazo possível, permitindo o 
tratamento antes da 24aa à 28르 semana gestacional, quando é mais efetivo para o feto; e ao manejo clínico adequado da gestante e seu(s) parceiro(s), incluindo o aconselhamento sobre a doença e formas de prevenção. Assim, poderá haver aumento da adesão ao tratamento e redução da vulnerabilidade das mulheres e seus parceiros às ISTs (BLENCOWE et al., 2011).

A ampliação da notificação dos casos de sífilis na gestação no SINAN, a busca sistemática de casos de sífilis congênita em todos os sistemas de informação e a melhoria do preenchimento das fichas de notificação e investigação são fundamentais para o melhor controle da doença (DOMINGUES et al., 2013).

Para mudar essa situação, o Departamento tem envidado esforços tais como: articulação com o Conselho Federal de Enfermagem, no sentido de sensibilizar os profissionais de enfermagem para a aplicação da penicilina durante o pré-natal; elaboração do Caderno de Boas Práticas de Sífilis; disponibilização do Protocolo de Investigação de Transmissão Vertical; articulação com o Conass e com o Conasems; elaboração do Protocolo Clínico e Diretrizes Terapêuticas (PCDT) para Atenção Integral às pessoas com Infecções Sexualmente Transmissíveis; interlocução com os laboratórios fabricantes da penicilina e com a ANVISA para solução dos problemas de desabastecimento. Essas estratégias têm-se mostrado adequadas e apontam que o Brasil caminha para o alcance de dupla eliminação da transmissão vertical do HIV e sífilis (BRASIL, 2015b).

\section{CONSIDERAÇÕES FINAIS}

Esta pesquisa ao traçar o perfil epidemiológico da sífilis congênita no município de Criciúma, constatou-se os óbitos fetais causados por sífilis congênita entre os anos de 2015 e 2016 encontrou-se um percentual de recém-nascidos natimortos de 3,7\% e vivos 96,3\%.

As Características do RN com Sífilis Congênita deu-se 96,3\% nascidos vivos e diagnosticados com Sífilis Congênita, 51,9\% foram caracterizados assintomáticos, 3,8\% sintomáticos e $42,3 \%$ não foi identificado nenhuma característica, sendo registrados como ignorados.

Quanto aos sinais e sintomas dos RN com sífilis congênita, 92,8\% dos dados não constavam os sinais ou sintomas e foram registrados como ignorados, sendo $3,6 \%$ apresentaram hepatomegalia e 3,6\% lesões cutâneas.

No que diz respeito ao diagnóstico da sífilis materna $66,6 \%$ das mulheres pesquisadas foram diagnosticadas ainda no pré-natal, sendo $29,6 \%$ diagnosticadas somente no parto ou na curetagem e apenas 3,7\% não foram encontradas nenhuma característica, registrando como ignorado.

Para tanto, conclui-se que a realização de pré-natal foi realizado por $92,6 \%$ das gestantes e apenas 7,4\% não realizaram o consultas de pré-natal. Os resultados deste estudo permitiu analisar o perfil da Sífilis Congênita e com isso evidenciou se que os 
objetivos da pesquisa foram alcançados.

A presente pesquisa mostra ainda dados que a incidência de sífilis congênita tem aumentado no Brasil, com mortalidade perinatal relevante e não há nenhuma expectativa de controle, mantendo-se as mesmas estratégias fracassadas das últimas décadas. Talvez a atualização dos conhecimentos técnicos, imposta pelas novas propostas para diagnóstico da sífilis, e a responsabilidade de realização do teste rápido nas unidades de saúde nos casos indicados exijam maior compromisso dos serviços e dos profissionais no controle da infecção congênita. A melhor estratégia é enfrentar este problema do passado com armas do futuro, que acaba de virar presente nas recomendações técnicas de diagnóstico da sífilis.

De acordo com a Portaria nำ3161 de 27 de Dezembro de 2011 do Ministério da Saúde, o tratamento com penicilina em gestantes deve ser iniciado sem nenhuma hesitação por parte da equipe de saúde, podendo ser aplicado 32 também nas unidades básicas de saúde e não só no âmbito hospitalar, as prescrições de penicilinas devem seguir os protocolos vigentes e o Formulário Terapêutico Nacional e a Relação Nacional de Medicamentos Essenciais (RENAME). O fornecimento da penicilina benzatina às unidades de saúde deve ser garantido e ininterrupto; caso haja problemas no abastecimento, a sua utilização para o tratamento de gestantes com sífilis deve ser priorizado (BRASIL, 2015b).

Para promover a melhoria dos casos de sífilis, os profissionais de saúde, principalmente o enfermeiro, devem participar ativamente na realização de atividades de educação em saúde que abordem e incentivem as formas de prevenção da doença, realizando todo o fluxo de ações preconizado pelo Ministério da Saúde, desde o diagnóstico precoce de sífilis em mulheres em idade reprodutiva até a notificação de todos os casos de sífilis congênita.

Segundo resoluções do Ministério da Saúde em 2014 (BRASIL, 2015c), por meio da Secretaria de Atenção a Saúde (SAS) e da Secretaria de Vigilância em Saúde (SVS) recomenda às Secretarias Estaduais de Saúde (SES) e Secretarias Municipais de Saúde (SMS), que adotem medidas que facilitem o acesso dos usuários do SUS ao diagnóstico da sífilis por meio dos testes rápido, sobretudo para as gestantes e seu parceiro, tais como:

- Ofertar teste rápido para sífilis em todas as Unidades Básicas de Saúde;

- Proporcionar as articulações e medidas locais necessárias que garantem a logística (acondicionamento, distribuição e transporte) e a execução do teste rápido nas UBS, com qualidade e confiabilidade;

- Avaliar a capacidade instalada laboratorial para a realização de exames complementares e de monitoramento do tratamento e de cura, com capacidade de entrega dos resultados, em até 7 dias;

- Planejar as capacitações em rede num processo de educação permanente;

- Utilizar os sistemas de Informação para registro da realização dos testes rápidos como SISPRENATAL WEB. 
Ainda que a sífilis seja uma patologia conhecida há séculos e que tenha agente etiológico bem definido, de fácil detecção, tratamento de baixo custo e cem por cento eficazes, ainda assim é considerado um grave problema de saúde pública que vem aumentando drasticamente principalmente no grupo de gestantes e 33 neonato. Estes dados instigam não só os gestores de saúde, mas também toda a humanidade, sendo, portanto difícil de ser eliminada. Apesar de grave, a sífilis é uma doença curável e não deixa sequelas, quando diagnosticada no início e tratada de forma ajustada, com acompanhamento de uma equipe qualificada que utilize de normas que visem o rastreio sistemático e a terapêutica adequada nas Unidades Básicas de Saúde; além de utilizar de ações de orientação sexual e de planejamento familiar na sua rotina de atendimento.

Os resultados reforçam que a redução da ocorrência da sífilis, somente será possível quando forem adotadas medidas mais efetivas de prevenção e controle. Neste contexto, são de suma importância o papel do enfermeiro na detecção da doença através da testagem rápida e acompanhamento do tratamento dos casos positivos.

Sugere-se ainda que mais pesquisas sejam realizadas sobre o tema para melhor delinear o perfil epidemiológico da sífilis congênita, disseminado conhecimentos a ponto de tornar o maior desafio na detecção precoce da sífilis materna e neonatal, o acompanhamento e tratamento uma realidade tangível e não longínqua.

\section{REFERÊNCIAS}

AZEVEDO, Jacinta. Infecções sexualmente transmissíveis. 30 Anos da Revista, 2008

BLENCOWE, Hannah et al. Lives Saved Tool supplement detection and treatment of syphilis in pregnancy to reduce syphilis related stillbirths and neonatal mortality. BMC public health, v. 11, n. 3, p. S9, 2011.

BRASIL. Ministério da saúde. Secretaria de vigilância em saúde - departamento de dst, aids e hepatites virais. Boletim epidemiológico - sífilis 2015a, ano iv- no 1 .

. Ministério da Saúde. Secretaria de Vigilância em Saúde. Departamento de DST, AIDS e Hepatites Virais. Caderno de boas práticas: o uso da penicilina na Atenção Básica para a prevenção da sífilis congênita no Brasil / Ministério da Saúde, Secretaria de Vigilância em Saúde, Departamento de DST, Aids e Hepatites Virais. - Brasília: Editora do Ministério da Saúde, 2015b.

. Ministério da Saúde. Secretaria de Vigilância em Saúde. Saúde Brasil 2014: uma análise da situação de saúde e das causas externas. Brasília: Ministério da Saúde, 2015c.

Ministério da saúde. Secretaria de vigilância em saúde - departamento de dst, aids e hepatites virais. Boletim epidemiológico - sífilis 2016, ano v- no 35 .

Ministério da Saúde. Secretaria de Vigilância em Saúde. Departamento de Vigilância Epidemiológica. Doenças infecciosas e parasitárias: guia de bolso/ 
Ministério da Saúde, Secretaria de Vigilância em Saúde, Departamento de Vigilância Epidemiológica. 8. ed. rev. Brasília: Ministério da Saúde; 2010. 448 p. (Série B. Textos Básicos de Saúde).

Ministério da Saúde. Secretaria de Vigilância em Saúde. Programa Nacional de DST/AIDS. Protocolo para prevenção de transmissão vertical de HIV e sífilis: manual de bolso. Brasília: Ministério da Saúde; 2007. 190 p. 35.

Ministério da Saúde. Secretaria de Vigilância em Saúde. Programa Nacional de DST/AIDS. Diretrizes para controle da sífilis congênita: manual de bolso /Ministério da Saúde, Secretaria de Vigilância em Saúde, Programa Nacional de DST/Aids. -2. ed. - Brasília : Ministério da Saúde, 2006.

.Conselho Nacional de Saúde. Resolução no 466, de 12 de dezembro de 2012, Disponível em: <http://conselho.saude.gov.br/resoluções/2012/Reso.pdf>. Acesso em 12/11/2017.

CARRARA, Sérgio. Tributo a Vênus: a luta contra a sífilis no Brasil, da passagem do século aos anos 40. SciELO-Editora FIOCRUZ, 1996.

CAMARGO JR, Kenneth R. de; COELI, Cláudia M. Reclink: an application for database linkage implementing the probabilistic record linkage method. Cadernos de saúde pública, v. 16, n. 2, p. 439-447, 2000.

DOMINGUES, Rosa Maria Soares Madeira et al. Sífilis congênita: evento sentinela da qualidade da assistência pré-natal. Revista de Saúde Pública, v. 47, n. 1, p. 147-157, 2013.

LABBE, Annie-Claude et al. The impact of syphilis, HIV-1, and HIV-2 on pregnancy outcome in Bissau, Guinea-Bissau. Sexually transmitted diseases, v. 29, n. 3, p. 157167, 2002.

LORENZI, Dino Roberto Soares; FIAMINGHI, Luciane Carvalho; ARTICO, Graziela Rech. Transmissão vertical da sífilis: prevenção, diagnóstico e tratamento. Femina, p. 83-90, 2009.

MAGALHÃES, Daniela Mendes dos Santos et al. Sífilis materna e congênita: ainda um desafio. Cadernos de Saúde Pública, p. 1109-1120, 2013.

ORGANIZAÇÃO MUNDIAL DA SAÚDE. Diagnóstico laboratorial de doenças sexualmente transmissíveis, incluindo o vírus da imunodeficiência humana. Brasilia: Ministério da Saúde, 2015.

SARACENI, Valéria; LEAL, Maria do Carmo. Avaliação da efetividade das campanhas para eliminação da sífilis congênita na redução da morbi-mortalidade perinatal. Município do Rio de Janeiro, 1999-2000. Cad Saúde Pública, v. 19, n. 5, p. 1341-9, 2003.

SARACENI, Valéria et al. Perinatal mortality due to congenital syphilis: a quality-ofcare indicator for women's and children's healthcare. Cadernos de saúde pública, v. 21, n. 4, p. 1244-1250, 2005.

STEER, Christopher et al. Spontaneous abortion rates after natural and assisted 
conception. BMJ: British Medical Journal, v. 299, n. 6711, p. 1317, 1989.

VALDEMARRA, Julia et al. Maternal syphilis and congenital syphilis in Latin America: big problem, simple solution. Revista Panamericana de Salud Pública, v. 16, n. 3, p. 209210, 2004.

TEMMERMAN, Marleen et al. Rapid increase of both HIV-1 infection and syphilis among pregnant women in Nairobi, Kenya. Aids, v. 6, n. 10, p. 1181-1186, 1992.

TRIDAPALLI, Elisabetta et al. Congenital syphilis in Italy: a multicentre study. Archives of Disease in Childhood-Fetal and Neonatal Edition, v. 97, n. 3, p. F211-F213, 2012. 